\title{
Prosthodontic Rehabilitation of Hypodontia - A Team Approach
}

\author{
Kiran Tariq ${ }^{1}$ \\ Muhammad Waseem Ullah Khan ${ }^{2}$ \\ Momina Akram ${ }^{3}$ \\ Sahar Illyas ${ }^{4}$ \\ Muhammad Azeem ${ }^{5}$ \\ BDS \\ BDS, FCPS \\ BDS, FCPS \\ BDS \\ BDS
}

Hypodontia can have an impact on speech, aesthetics, function and psycho-social behavior of individuals afflicted by the problem. Rehabilitation of patients with hypodontia usually requires complex treatment planning, depending upon the pattern of tooth absence, amount of residual spacing, presence of malocclusion and patient compliance. It is an interdisciplinary intervention, involving prosthodontists, orthodontists, oral surgeons, speech pathologists and psychologists to achieve an optimal outcome for the patient. This case report describes the close work of a committed team from orthodontic, oral surgery and prosthodontic department of de'Montmorency College of Dentistry, Lahore, to reach final aesthetic and functional outcomes for an 18 year old girl with oligodontia. Her chief complaint was poor esthetics and absence of multiple anterior teeth with unsightly spacing between remaining teeth. Orthodontic redistribution of spaces followed by fixed full arch prosthetic rehabilitation were the goals achieved at the end of her treatment.

KEYWORDS: Oligodontia, interdisciplinary team work, prosthodontic rehabilitation, fixed restorations.

HOW TO CITE: Tariq K, Khan MWU, Akram M, Illyas S, Azeem M. Prosthodontic rehabilitation of hypodontia- A team approach. J Pak Dent Assoc 2021;30(4):279-284.

DOI: https://doi.org/10.25301/JPDA.304.279

Received: 22 October 2020, Accepted: 14 July 2021

\section{INTRODUCTION}

$\mathrm{O}$ ne of the most common dentofacial malformation in humans is hypodontia. ${ }^{1}$ It is characterized by agenesis or developmental failure of dental germ to fully develop and erupt into final dentition. In more advance form hypodontia may present as oligodontia or anodontia, resulting in the absence of more than six to eight teeth or the absolute dentition. ${ }^{2}$ Women are more afflicted with this variation than men, and its prevalence ranges from $0.08 \%$ to $1.55 \%$ in primary dentition and $2.3 \%$ to $11.3 \%$ in secondary dentition, respectively. ${ }^{3,4}$ Hypodontia may be associated with recognized genetic syndromes involving growth and developmental defects, for instance Down's syndrome, Crouzon's syndrome and Ectodermal dysplasia5.

1. FCPS resident, Department of prosthodontics, de'Montmorency College of Dentistry, Lahore.

2. Assistant Professor, Department of Prosthodntics, de'Montmorency College of Dentistry, Lahore.

3. Assistant Professor, Department of Prosthodontics, de'Montmorency College of Dentistry, Lahore.

4. MDS resident, Department of Orthodontics, de'Montmorency College of Dentistry, Lahore.

5. FCPS resident, Department of Oral and Maxillofacial surgery, de'Montmorency College of Dentistry, Lahore.

Corresponding author: "Dr. Kiran Tariq" < kirantariq1234@gmail.com >
It can also occur due to isolated non-syndromic genetic inheritance as a polygenic defect with point mutations in MSX1 gene (missing second premolars), PAX9 gene (missing upper lateral incisors) and AXIN2 gene (missing lower incisors). ${ }^{6,7}$

Clinically, individuals with hypodontia have teeth that are generally smaller than normal- microdonts, and often have more simplified shapes. These teeth usually have enamel hypoplasia, shortened roots and ectopic positioning. Teeth are often rotated and normal occlusion is frequently disrupted. Skeletal patterns in hypodontia patients present with less than normal mandibular plane angles in association with decreased lower anterior facial height and a tendency towards skeletal Class III. As the number of missing teeth increases (more than six), these patterns become more prominent. ${ }^{8}$ Patients with hypodontia may present at early mixed dentition, late mixed/early permanent and late permanent dentition. Treatment planning for mixed dentition includes prevention and reassurance, removable dentures/overdentures for psychological and functional reasons, composite build ups to improve aesthetics of microdonts, simple orthodontic space closures and schematic extractions of retained primary teeth to guide permanent tooth eruptions. ${ }^{9}$ Permanent dentition treatment planning aims at more definitive stabilization of 
occlusion with comprehensive orthodontic treatment and permanent bridge work including implant supported prosthesis after growth completion. ${ }^{10}$

Hypodontia can range in complexity from simple to severe forms. Simple cases are often straight forward to treat. They present with one or two missing teeth especially upper lateral incisors with usually adequate space available to restore with resin retained bridges or implants. ${ }^{11}$ Cases with crowding and drifting of adjacent teeth into missing tooth spaces add complexity in treatment planning and demand referral for orthodontic repositioning of teeth to create space followed by prosthetic restorations. Retained deciduous teeth may pose problems with eruption pattern/timing of permanent teeth and pediatric dentists are required to schedule extractions and guide eruptions of impacted permanent teeth. Severe hypodontia presents with multiple missing teeth in all quadrants, retained deciduous teeth, inadequate restorative space, loss of occlusal vertical dimension and disparities in occlusal plane. Targeting an ideal occlusion may not be possible in these cases and presents the most challenging scenario. Treatment planning includes multidisciplinary approach from different specialties to plan orthodontic tooth movements to optimize interdental spaces and occlusal plane, establishing occlusal vertical dimension for prosthetic restorations and sinus lift/bone augmentation surgeries to plan implant bridge-works. ${ }^{12,13}$

Multidisciplinary coordination among different specialties is important in treatment planning of individuals with hypodontia. It enables to provide an optimum care for patient in a scheduled way according to the complexity of presentation. It is an interdisciplinary intervention, involving prosthodontists, orthodontists, oral surgeons, pediatric dentists, speech pathologists and psychologists to achieve an optimal outcome for the patient. The purpose of this case report is to describes the close work of a committed team from orthodontic, oral surgery and prosthodontic department of de'Montmorency College of Dentistry, Lahore, to reach final aesthetic and functional outcomes for a patient with oligodontia.

\section{CASE REPORT}

An 18-year-old girl presented to the Outpatient Department of Prosthodontics, de'Montmorency College of Dentistry, Lahore. Her chief complaint was poor esthetics caused by absence of multiple anterior teeth and unsightly spacing between remaining teeth. Detailed medical and dental history revealed that no previous dental treatment was received by the patient and medical history was insignificant. Family history revealed that one of her younger sibling also had the same dental problem. This finding highlighted the hereditary pattern of hypodontia.

During extra oral examination, no classical syndromic features were found in facial appearance. She had symmetric face with straight profile and normal hair, eyes, ears and nose. Lips were competent with normal perioral musculature. Lower anterior facial height was reduced with decrease in vertical dimension of occlusion. Temporomandibular joint examination revealed no abnormality in function or associated pain in muscles of mastication.

During intraoral examination, multiple permanent teeth were missing, (Figure 1a). Few deciduous teeth were retained with grade 2 mobility (Millers classification) 14, these were maxillary primary canines and the mandibular left primary canine.

Figure 1a: Pre-op intra oral view

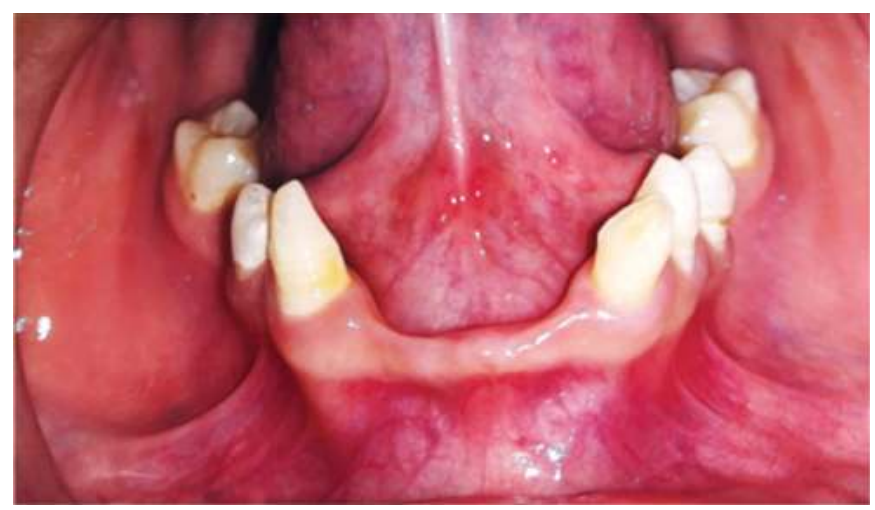

Figure 1b: Pre-op intra oral view

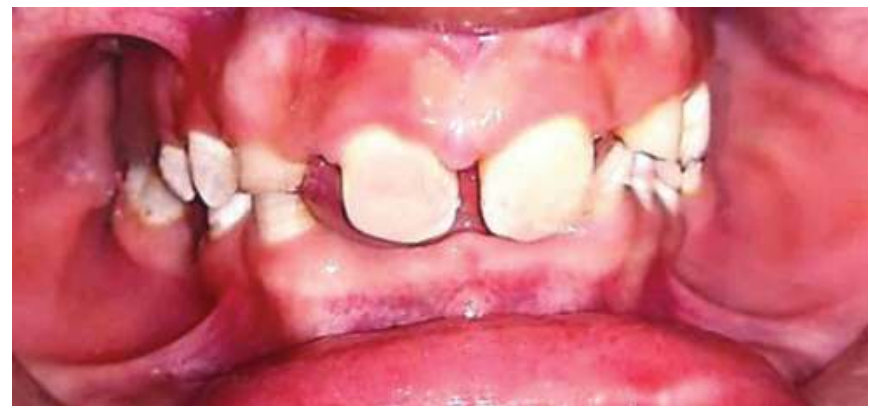

Maxillary central incisors were spaced with a midline diastema and were the most prominent teeth in the arch. Their incisal plane was near the crest of the mandibular anterior ridge, which was edentulous from canine to canine and narrow buccolingually (figure 1b). Remaining teeth were small and irregular in shape- microdonts (figure 1c, 1d). Anterior guidance was lost. Reduced occlusal vertical dimension with increased freeway space of up to $6 \mathrm{~mm}$ was recorded. Regarding soft tissue status, oral mucosa, tongue and gingiva were normal in color and texture and oral hygiene status was satisfactory. Maxillary labial frenal attachment was low and thick. Panoramic radiograph evaluation revealed resorbed roots of retained primary teeth and absence of any 


Tariq K/ Khan MWU/ Akram M/
Illyas S/ Azeem M

Prosthodontic rehabilitation of hypodontia - A team approach

Figure 1c:

Pre-op maxillary cast

Figure 1d:

Pre-op mandibular cast
Figure 2a: Pre-op Orthopantomogram

Figure 2b: Pre-op

Lateral cephalogram
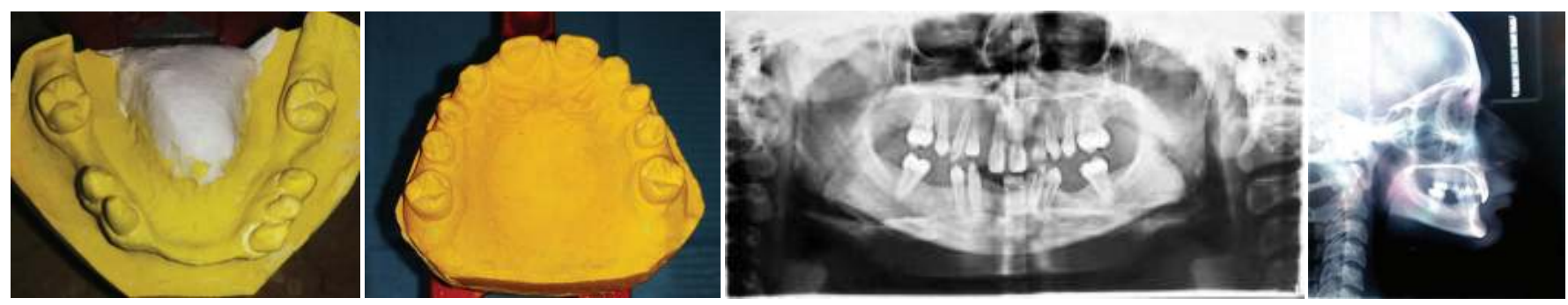

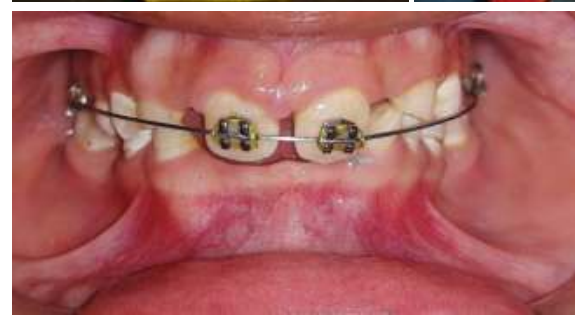

Figure 3a: Leveling and alignment

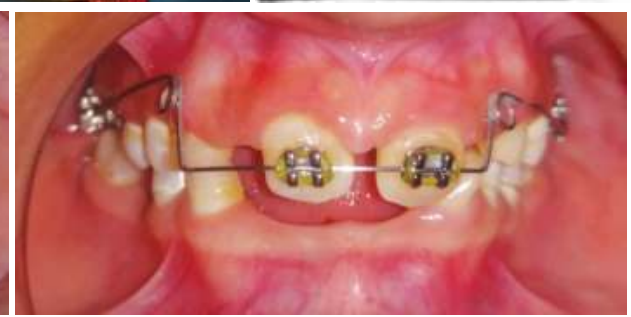

Figure 3b: Protraction and intrusion of central incisors

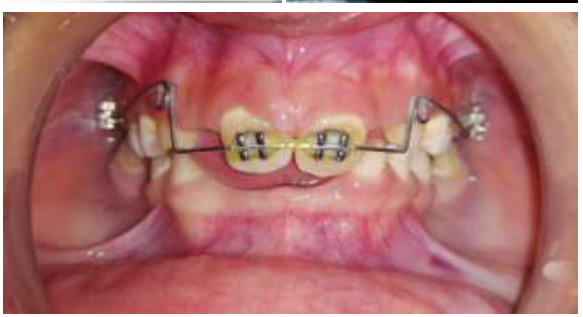

Figure 3c:

Diastema closure impacted or unerupted permanent tooth inside the jaws (figure 2a). On lateral cephalogram (figure 2b) skeletal class 1 was confirmed with low mandibular plane angle and deep bite. Upper inclinations were retroclined.

After a thorough clinical and radiographic examination, Oligodontia was diagnosed. Multidisciplinary treatment approach was planned to reach the final treatment outcomes. Diagnostic casts were obtained using alginate hydrocolloid impressions for both upper and lower arches. Possible treatment options were worked out in a written form with an explanation of advantages and disadvantages for each option. A detailed consultation with the patient about the ideal to least ideal treatment plan was undertaken as follow:

1. Orthodontic treatment to correct alignment and spacing of upper central incisors, restoring height and shape of microdonts with individual crowns and replacement of missing teeth with osseointegrated implants.

2. Orthodontic treatment to correct alignment and spacing of upper central incisors and restoring missing teeth using fixed partial dentures (porcelain fused to metal bridges) in upper and lower arches.

3 . Restoring the missing teeth using overlay type removable partial prosthesis.

Due to financial constraints and esthetic demands, the patient opted for treatment plan number 2. A multidisciplinary team from departments of orthodontics, oral surgery and prosthodontics was involved to initiate the treatment. Before commencement of treatment, a written in-formed consent was taken from the patient for the publication of this case report, and the patient refused to give consent to publish extraoral photographs.

\section{Adjunctive orthodontics}

First step was a consultation with an orthodontist for the correction of alignment and spacing of maxillary central incisors. Orthodontic repositioning of maxillary central incisors was planned. It was scheduled in three phases: Phase 1: leveling and alignment (figure 3a)

Phase 2: intrusion and proclination of central incisors using protraction and intrusion utility arch wire (figure $3 \mathrm{~b}$ ) Phase 3: closure of midline diastema (figure 3c)

A six month duration was assigned to complete adjunctive orthodontics.

\section{Pre-Prosthetic Surgery}

Second step was pre-prosthetic surgery by an oral surgeon. Following goals were achieved after pre -prosthetic surgery:

1: Frenectomy of thick upper labial frenum to prevent relapse after orthodontic closure of midline diastema (figure 4a)

2: Surgical crown lengthening with gingivectomy around central incisors (figure $4 \mathrm{~b}$ )

3: Extraction of retained deciduous teeth with resorbed roots and clinical grade 2 mobility (upper primary canines and lower left primary canine.)

\section{Prosthetic rehabilitation}

Final step was the restorative phase involving prosthetic rehabilitation. It was done in two stages: laboratory phase and clinical phase. 


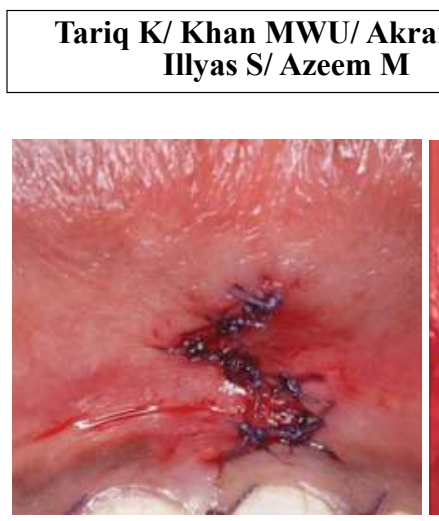

Figure 4a:

Post frenectomy

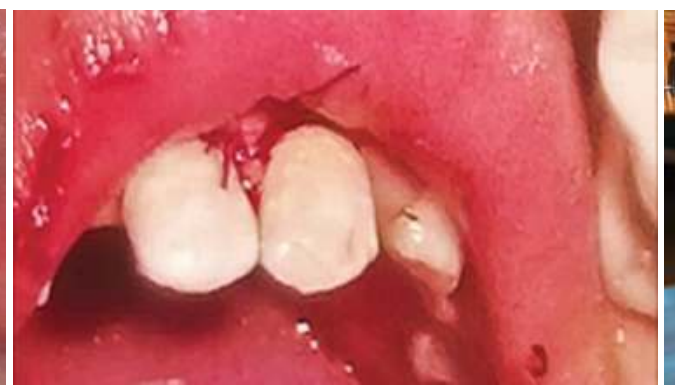

Figure 4b:

Post gingivectomy

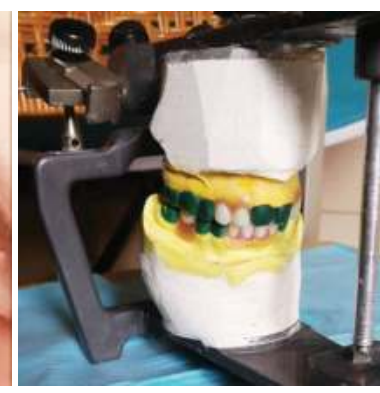

Figure 5c: Diagnostic wax-up (right side)

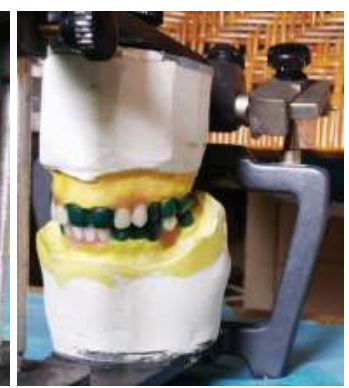

Figure 5d: Diagnostic wax-up (left side)

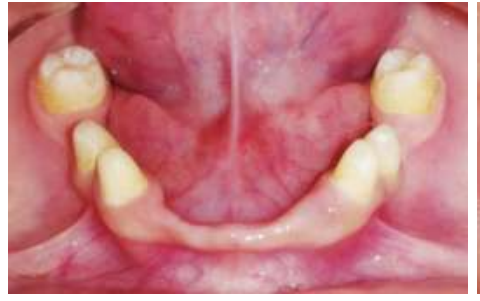

Figure 6a: Mandibular arch tooth preparations

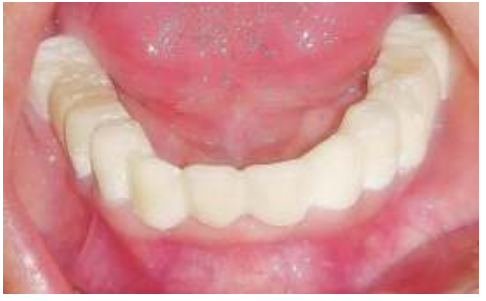

Figure 6b: Cemented Lower arch provisional prosthesis

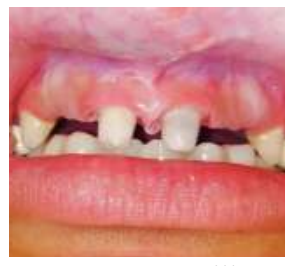

Figure 7a:Maxillary arch tooth preparations, central incisors prepared conventionally

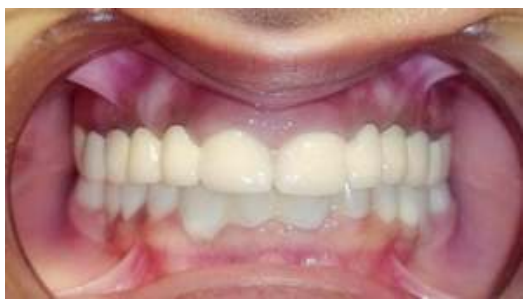

Figure 7b: Cemented Upper arch provisional prosthesis.

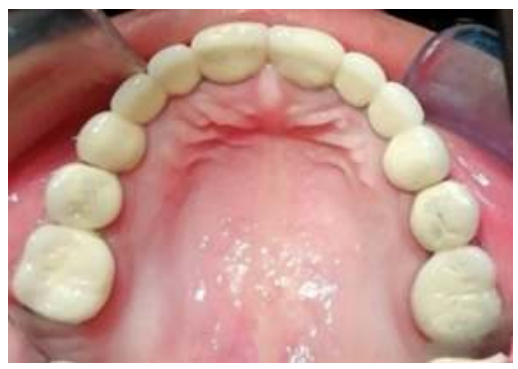

Figure 8a: Definitive Upper arch porcelain fused to metal prosthesis

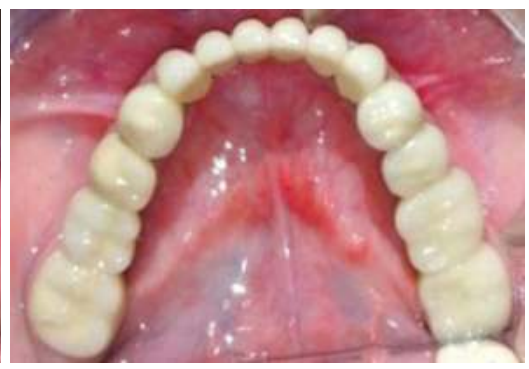

Figure 8b: Definitive lower arch porcelain fused to metal prosthesis

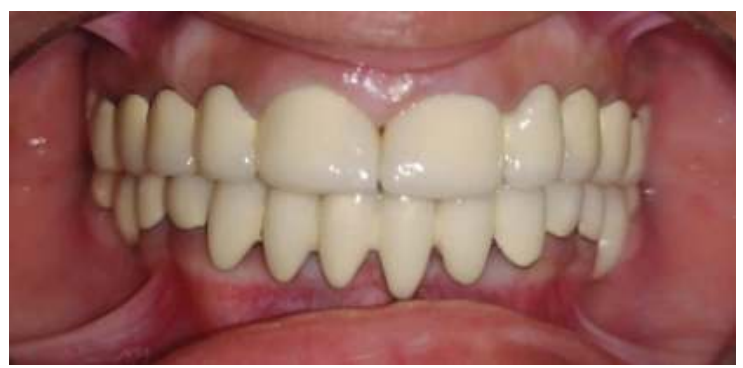

Figure 8c: Final prosthesis in occlusion

\section{Laboratory phase}

Diagnostic impression for both maxillary and mandibular arches was taken in irreversible hydrocolloid impression material. Discrepancy between resting vertical dimension and occlusal vertical dimension (OVD) was recorded on patient. A freeway space of $6 \mathrm{~mm}$ was found to be present. Face bow transfer record was taken and the diagnostic casts were articulated on semi-adjustable Arcon type articulator (Bio-Art) in centric jaw relationship. Diagnostic wax-up was done on articulated casts (figure 5a, 5b). Occlusal vertical height was established with diagnostic wax-up with a total of $4 \mathrm{~mm}$ increase in occlusal vertical dimension ( $2 \mathrm{~mm}$ increase for each arch). Full arch segmented fixed partial dentures (porcelain fused to metal bridges) at established occlusal vertical dimension for both upper and lower arches were decided upon as final treatment outcome. Diagnostic wax up was translated to provisional fixed acrylic bridges in the lab.

\section{Clinical phase}

Tooth preparation for microdonts was done conservativelymargins were defined, undercuts and sharp line angles were removed and axial walls were made parallel. Only upper central incisors, which were larger in size, were reduced conventionally for porcelain fused to metal restorations preceded with elective endodontic. Mandibular arch teeth were prepared first and provisional restoration was delivered at a $2 \mathrm{~mm}$ increase in occlusal vertical dimension (figure $6 \mathrm{a}$, $6 b)$. After 2 weeks, patient was recalled and her adaptation at increased occlusal vertical dimension was evaluated. Maxillary arch teeth were prepared and upper provisional restoration at $2 \mathrm{~mm}$ increased occlusal vertical dimension was delivered (figure 7a,7b). Patient was kept on a two weekly follow-up for next 2 months, at every visit her adaptation at established occlusal vertical dimension was evaluated. After 2 months, no pain or difficulty in function was reported and temporomandibular joint examination was 
also normal. Final impression for tooth preparations was taken with putty wash technique in addition silicon elastomeric impression material. Jaw relation records were repeated and sent to the laboratory for the fabrication of definitive porcelain fused to metal bridges at an established occlusal vertical dimension. Before final delivery of the prosthesis, an appointment for metal casting try-in was also scheduled. Definitive prosthesis was cemented with glass ionomer luting cement (figure 8a, 8b, 8c).

\section{DISCUSSION}

The treatment objective for this case was to create a fair starting point for prosthodontic rehabilitation. This was mainly accomplished by orthodontic intrusion and proclination of upper central incisors. Orthodontic tooth movements of maxillary central incisors were essential because these were larger than the remaining microdonts and occlusal plane was disturbed due to their size and supra eruption against edentulous mandibular anterior ridge. Reduction in overbite must be taken into consideration whenever restoration of space is planned anteriorly. ${ }^{15}$ Moreover, midline diastema closure created favorable spaces distal to central incisors for future prosthesis. Labial frenectomy was done to prevent relapse of midline diastema. Loss of occlusal vertical dimension (OVD) is a common manifestation among patients with multiple missing teeth and its re-establishment by fixed or removable prosthesis is the most challenging and a complex procedure. For this reason an initial adaptation period with a provisional prosthesis at appropriate occlusal vertical height is required before final restoration. ${ }^{16}$ This allows a prudent assessment of patient's tolerance, functioning and esthetics at the proposed occlusal vertical dimension. An increase of upto $5 \mathrm{~mm}$ in occlusal vertical dimension is an achievable alteration, as physiologic OVD occurs at a range, known as the comfort zone, rather than a specified value. ${ }^{17}$ The patient can adapt to a change in OVD as long as it lies in this range. This case presents a $4 \mathrm{~mm}$ increase in occlusal vertical height using provisional fixed restorations for a 6-8 weeks adaptation period. Follow-up after 8 weeks revealed neither symptoms nor any temporomandibular joint dysfunction.

Conventional fixed prosthodontics depends on preparing healthy teeth next to the missing ones, and often require elective endodontics in teeth with large pulp chambers. ${ }^{18}$ However, in this case, where a $2 \mathrm{~mm}$ increase in OVD was planned per arch, need for excessive crown preparations was precluded, and tooth preparations were kept as minimal as possible for microdonts, only sharp line angles were removed, margins were defined and parallelism was established.
Treatment of hypodontia may require that osseointegrated dental implants be used to replace missing teeth. In this clinical case, a conservative approach was selected without the need for implants. ${ }^{19}$ There is limited literature to address long term results for aesthetic, functional and satisfaction levels among hypodontia patients restored with dental implants. ${ }^{20}$ Adequate alveolar bone and keratinized gingival biotype are prerequisite to successful implant insertion. However, reduced buccolingual width and apico-coronal height of alveolar ridges in patients with hypodontia, warrants bone augmentation before any dental implant placement. ${ }^{21}$ Therefore, finances and time required to undergo extensive implant surgeries poses a limitation to patient's preference to this treatment modality.

Patients presenting with few missing teeth can be treated by individual dental implants or minimal preparation fixed prosthesis. However for patients with multiple missing teeth, treatment options may range from osseointegrated implants and fixed prosthesis to overdentures and temporary/definitive removable partial dentures.

\section{CONCLUSION}

There is an array of treatment modalities to dental rehabilitation of patients with hypodontia. However, early prosthetic intervention can improve masticatory function, speech, esthetics and self-esteem among these individuals. Early diagnosis and continuous dedication of preliminary management teams is important in the overall treatment plan. Well planned restorative approach in this case has contributed to achieve optimum final treatment outcomes.

\section{CONFLICT OF INTEREST}

None to declare

\section{REFERENCES}

1. Laganà G, Venza N, Borzabadi-Farahani A, Fabi F, Danesi C, Cozza P. Dental anomalies: prevalence and associations between them in a large sample of non-orthodontic subjects, a cross-sectional study. BMC Oral Health. 2017;17:62.

https://doi.org/10.1186/s12903-017-0352-y

2. Al-Ani AH, Antoun JS, Thomson WM, Merriman TR, Farella M. Hypodontia: An Update on Its Etiology, Classification, and Clinical Management. Biomed Res Int. 2017;2017:9378325.

https://doi.org/10.1155/2017/9378325

3: Jepson NJ, Nohl FS, Carter NE, Gillgrass TJ, Meechan JG, Hobson RS, Nunn JH. The interdisciplinary management of hypodontia: restorative dentistry. Br Dent J. 2003;194:299-304.

https://doi.org/10.1038/sj.bdj.4809940 
4. Gracco ALT, Zanatta S, Forin Valvecchi F, Bignotti D, Perri A, Baciliero F. Prevalence of dental agenesis in a sample of Italian orthodontic patients: an epidemiological study. Prog Orthod. 2017;18:33.

https://doi.org/10.1186/s40510-017-0186-9

5. Rakhshan V. Congenitally missing teeth (hypodontia): A review of the literature concerning the etiology, prevalence, risk factors, patterns and treatment. Dent Res J. 2015;12:1-13. https://doi.org/10.4103/1735-3327.150286

6. Tangade SP, Ravishankar TL, Batra M, Shah AF. Familial Hypodontia: A Case Series. Kathmandu Univ Med J. 2015;13:1679.

https://doi.org/10.3126/kumj.v13i2.16792

7. Pemberton TJ, Das P, Patel PI. Hypodontia: genetics and future perspectives. Braz J Oral Sci, 2015;4:695-706.

8. Taju W, Sherriff M, Bister D, Shah S. Association between severity of hypodontia and cephalometric skeletal patterns: a retrospective study. Eur J Orthod. 2017;40:200-05.

https://doi.org/10.1093/ejo/cjx049

9. Tong HJ, Tahmassebi JF. Management of a child with severe hypodontia in the mixed dentition stage of development. Eur Arch Paediatr Dent. 2014;15:449-54.

https://doi.org/10.1007/s40368-014-0137-9

10. Hvaring CL, Øgaard B, Birkeland K. Tooth replacements in young adults with severe hypodontia: Orthodontic space closure, dental implants, and tooth-supported fixed dental prostheses. A follow-up study. Am J Orthod Dentofacial Orthop. 2016;150:62026.

https://doi.org/10.1016/j.ajodo.2016.03.023

11. Hobson RS, Carter NE, Gillgrass TJ, Jepson NJ, Meechan JG, Nohl F, Nunn JH. The interdisciplinary management of hypodontia: the relationship between an interdisciplinary team and the general dental practitioner. Br Dent J. 2003;194:479-82.

https://doi.org/10.1038/sj.bdj.4810184

12. Harrison J. Management of patients with hypodontia: What has changed? J Orthod. 2019;46:60-64. https://doi.org/10.1177/1465312519840043
13. Attia S, Schaaf H, El Khassawna T, Malhan D, Mausbach K, Howaldt HP, Streckbein P. Oral Rehabilitation of Hypodontia Patients Using an Endosseous Dental Implant: Functional and Aesthetic Results. J Clin Med. 2019;8:1687.

https://doi.org/10.3390/jcm8101687

14. Wu CP, Tu YK, Lu SL, Chang JH, Lu HK. Quantitative analysis of Miller mobility index for the diagnosis of moderate to severe periodontitis - A cross-sectional study. J Dent Sci. 2018;13:43-47. https://doi.org/10.1016/j.jds.2017.11.001

15. Carter NE, Gillgrass TJ, Hobson RS, Jepson N, Eechan JG, Nohl FS, Nunn JH. The interdisciplinary management of hypodontia: orthodontics. Br Dent J. 2003;194:361-6. https://doi.org/10.1038/sj.bdj.4809995

16. Marin DO, Leite AR, de Oliveira Junior NM, Compagnoni MA, Pero AC, Arioli Filho JN. Reestablishment of Occlusal Vertical Dimension in Complete Denture Wearing in Two Stages. Case Rep Dent. 2015;2015:762914.

https://doi.org/10.1155/2015/762914

17. Abduo J. Safety of increasing vertical dimension of occlusion: a systematic review. Quintessence Int. 2012;43:369-80.

18. Sadaqah N, Tair J. Management of Patient with Hypodontia: Review of Literature and Case Report. Open J Stomatol. 2015;05:293308.

https://doi.org/10.4236/ojst.2015.512036

19. Filius MAP, Vissink A, Cune MS, Raghoebar GM, Visser A. Long-term implant performance and patients' satisfaction in oligodontia. J Dent. 2018;71:18-24.

https://doi.org/10.1016/j.jdent.2018.01.007

20. Burns B, Grieg V, Bissell V, Savarrio L. A review of implant provision for hypodontia patients within a Scottish referral centre. Br Dent J. 2017;223:96-99.

https://doi.org/10.1038/sj.bdj.2017.623

21. Bertl K, Grotthoff VS, Bertl MH, Heimel P, Gahleitner A, Ulm $\mathrm{C}$, Stavropoulos A. A wide mesio-distal gap in sites of congenitally missing maxillary lateral incisors is related to a thin alveolar ridge. Clin Oral Implants Res. 2017;28:1038-1045.

https://doi.org/10.1111/clr.12915 